\title{
Solution Properties of Synthetic Polypetides \\ VII. Approximate Expressions for Important Physical Quantities of Polypeptide Molecules
}

\author{
Akio Teramoto, Takashi Norisuye, and Hiroshi Fujita \\ Department of Polymer Science, Osaka University, Toyonaka, Osaka, Japan.
}

(Received July 16, 1969)

\begin{abstract}
Approximate expressions, which are amenable to straightforward numerical calculation, are derived from Nagai's theory for such important molecular quantities of polypeptides in solution as helical content $f_{N}$, number of helical sections $g_{N}$, mean-square radius of gyration $\left\langle S^{2}\right\rangle$, and particle scattering function $P(\theta)$, subject to the condition that $\sqrt{\sigma} \ll 1, N^{-1} \ll 1$, and $N \sqrt{\sigma}>3$. Here $N$ denotes the degree of polymerization of the polypeptide molecule and $\sigma$ the helix initiation parameter. The derived expressions are compared numerically with the exact ones for some typical cases of the parameters involved, and it is shown that they are accurate enough for practical purposes. A brief discussion is given on how each of them can be of use for characterizing polypeptides in dilute solution.
\end{abstract}

KEY WORDS Solution Properties / Statistical Theory /

Helix-Coil Transition / Synthetic Polypeptide / Helical Content /

Molecular Dimensions / Light Scattering Function /

Nagai $^{1}$ was the first to derive rigorous expressions for such important physical quantities of helix-forming polypeptide molecules in solution as the mean square radius of gyration, the meansquare end-to-end distance, the alternating frequency of helical and randomly coiled sections, and the particle scattering function. Three parameters $N, u$, and $\sigma$ appear commonly in all of these expressions.* Here $N$ is the degree of polymerization of the chain, $u$ is the activity for helix formation (or the statistical weight of a peptide residue present inside a helical section), and $\sigma$ is the helix initiation parameter ( $\sigma u$ represents the statistical weight of a peptide residue located at the boundary between helical and randomly coiled sections). Unfortunately, $\mathrm{Na}$ gai's results ${ }^{1}$ as well as those of subsequent authors $^{3,4}$ who dealt with similar problems are so complicated that not only are they formidable for numerical computation but it is practically impossible for them to be adapted for experimental data.

In Part $\mathrm{VI}^{2}$ of this series we have shown that

* To conform with our previous paper $^{2}$ (Part VI), the rather commonly used notation $s$ for the activity for helix formation is replaced here by $u$. Nagai ${ }^{1}$ used the symbol $\sigma$ for $u$ and the symbol $\alpha^{2}$ for $\sigma$. if we take account of the fact that, in actual systems, the parameter $\sigma$ is as small as of the order of $10^{-4}$ and if we confine ourselves to samples whose $N$ is large enough to make $N \sqrt{\sigma}$ larger than 3 , Nagai's expression for $f_{N}$ can be reduced to a very simple form readily applicable to the determination of $u$ and $\sigma$ experimentally. Here $f_{N}$ designates the helical content of a polypeptide sample whose degree of polymerization is $N$. The present paper attempts to derive approximate expressions for the above-mentioned physical quantities $\left(\left\langle S^{2}\right\rangle,\left\langle R^{2}\right\rangle\right.$, etc.) from the exact ones of Nagai under the same conditions for $\sigma$ and $N$, although an additional restriction has to be introduced for obtaining a relevant expression for the particle scattering function $P(\theta)$. Whenever possible, the accuracy of the derived expressions is examined numerically by comparing them with the exact ones for typical cases of the parameters involved, and their practical significance is briefly discussed in relation to the analysis or interpretation of experimental data on polypeptides in dilute solution.

\section{APPROXIMATE EXPRESSIONS}

\section{Nature of Approximations Used}

Basic to Nagai's theory ${ }^{1}$ cited above are the 


\section{A. Teramoto, T. Norisuye, and H. Fujita}

four eigenvalues $\lambda_{i}(i=1,2,3,4)$ which are determined as functions of $u$ and $\sigma$ by the equation

$$
\lambda_{i}{ }^{2}\left(\lambda_{i}-1\right)\left(\lambda_{i}-u\right)=\sigma u
$$

It has been shown in Part VI that when $\sqrt{\sigma} \ll 1, \lambda_{i}$ is expanded in powers of $\sqrt{\sigma}$ to give

$$
\begin{aligned}
& \lambda_{1}=1+\sqrt{\sigma}\left(z+\sqrt{1+z^{2}}\right)+0(\sigma) \\
& \lambda_{2}=1+\sqrt{\sigma}\left(z-\sqrt{1+z^{2}}\right)+0(\sigma) \\
& \lambda_{3}=\sqrt{\sigma}+0(\sigma) \\
& \lambda_{4}=-\sqrt{\sigma}+0(\sigma)
\end{aligned}
$$

where $z$ is defined by

$$
z=(\ln u) / 2 \sqrt{\sigma}
$$

When Eq. 2 is inserted into Nagai's expression for $f$, the value of $f_{N}$ for infinitely large $N$, it can be readily shown that

$$
f=(1 / 2)\left(1+z / \sqrt{1+z^{2}}\right)+0(\sigma)
$$

Solving this equation for $z$ gives

$$
z=(2 f-1) / 2 \sqrt{f(1-f)}+0(\sqrt{\sigma})
$$

which, upon introducing into Eqs. 2 and 3, yields

$$
\begin{aligned}
& \lambda_{1}=1+\sqrt{\sigma} \sqrt{\frac{f}{1-f}}+0(\sigma) \\
& \lambda_{2}=1-\sqrt{\sigma} \sqrt{\frac{1-f}{f}}+0(\sigma)
\end{aligned}
$$

Thus we see that the four eigenvalues in question are expressed in terms of $\sqrt{\sigma}$ and/or $f$. Ptitsyn and Skvortsev $^{5}$ have already derived Eq. 9 from the theory of Zimm and Bragg. ${ }^{6}$

In the subsequent development, when these expressions for $\lambda_{i}$ are substituted into Nagai's equations for various quantities such as $f_{N}$, $\left\langle S^{2}\right\rangle$, and so on and the results are expanded in powers of $\sqrt{\sigma}$, we drop terms multiplied by $\sqrt{\sigma}$ and its higher powers in comparison with unity. Moreover, terms of the order of $N^{-1}$ and its higher powers are ignored in comparison with unity by confining our interest to samples of sufficiently long chain. This does not mean, however, that terms of the order of $(N \sqrt{\sigma})^{-1}$ or its higher powers which appear quite often in the process of derivation are neglected. Since $\sigma$ is as small as $10^{-4}$, the product $N \sqrt{\sigma}$ remains not too large in comparison with unity even for $N$ as large as 500. Thus in order to obtain a better approximation it is important to retain terms which are multiplied by $(N \sqrt{\sigma})^{-1}$ and its higher powers.

Nagai's equations for the various quantities considered are written as a sum of terms which are proportional to $\lambda_{1}{ }^{N}, \lambda_{2}{ }^{N}, \lambda_{3}{ }^{N}, \lambda_{4}{ }^{N}$, and $u^{N}$. Therefore, the relative mangitudes of those terms in each equation are chiefly determined by that of a factor $\left(\lambda_{i} / \lambda_{1}\right)^{N}(i=1,2,3,4)$ or $\left(u / \lambda_{1}\right)^{N}$ relative to unity. It follows from Eqs. 2, 4, and 5 that the terms multiplied by $\left(\lambda_{3} / \lambda_{1}\right)^{N}$ and $\left(\lambda_{4} / \lambda_{1}\right)^{N}$ are proportional to $\sigma^{N / 2}$, and hence, under the conditions assumed above for $\sqrt{\sigma}$ and $N$, they can be totally ignored in comparison with unity. Subject to the same conditions for $\sqrt{\sigma}$ and $N$, the factor $\left(\lambda_{2} / \lambda_{1}\right)^{N}$ may be written

$$
\left(\lambda_{2} / \lambda_{1}\right)^{N}=\exp \left[-\frac{N \sqrt{\sigma}}{\sqrt{f(1-f)}}\right]+0(\sqrt{\sigma})
$$

Since this exponential function never exceeds $\exp (-2 N \sqrt{\sigma})$, the terms multiplied by $\left(\lambda_{2} / \lambda_{1}\right)^{N}$ may be taken to be negligible in comparison with unity, provided that, in addition to the abovementioned conditions for $\sigma$ and $N$, the product $N \sqrt{\sigma}$ is larger than 3 . In the subsequent development, therefore, we drop such terms by confining ourselves to the case that this restriction on $N \sqrt{\sigma}$ holds. It can be shown in a similar manner that for $\sqrt{\sigma} \ll 1$ and $N \gg 1$ the factor $\left(u / \lambda_{1}\right)^{N}$ may be written

$$
\left(u / \lambda_{1}\right)^{N}=\exp \left[-N \sqrt{\sigma} \sqrt{\frac{1-f}{f}}\right]+0(\sqrt{\sigma})
$$

This approaches unity as $f$ tends to unity however large the product $N \sqrt{\sigma}$ may be, and hence the terms multiplied by $\left(u / \lambda_{1}\right)^{N}$ must be retained in our development even if $N \sqrt{\sigma}$ is assumed to be larger than 3.

\section{Helical Content}

For this quantity we have already derived, in Part VI, ${ }^{2}$ the desired approximate expression subject to the above-mentioned conditions for $\sqrt{\sigma}, N$, and $N \sqrt{\sigma}$. It reads*

$$
f_{N}=f-\frac{2 f^{3 / 2}(1-f)^{1 / 2}}{\beta}
$$

* Eq. 13 of Part VI retains terms associated with the factor $\left(\lambda_{2} / \lambda_{1}\right)^{N}$, i.e., $\exp [-\beta / \sqrt{f(1-f)]}$ (see Eq. 11). 
Approximate Expressions for Important Physical Quantities of Polypeptide Molecules

Table I. Comparison of the exact and approximate expressions for the helical content $f_{N} ; \sigma$ is taken to be $2.0 \times 10^{-4}$

\begin{tabular}{|c|c|c|c|c|c|c|}
\hline \multirow{2}{*}{$z^{\mathrm{a}}$} & \multicolumn{2}{|c|}{$N=\infty$} & \multicolumn{2}{|c|}{$N=1200$} & \multicolumn{2}{|c|}{$N=600$} \\
\hline & exact & approx. & exact & approx. & exact & approx. \\
\hline-2.163 & 0.051 & 0.046 & 0.050 & 0.045 & 0.049 & 0.044 \\
\hline-1.570 & 0.085 & 0.078 & 0.082 & 0.076 & 0.079 & 0.073 \\
\hline-0.736 & 0.211 & 0.204 & 0.201 & 0.194 & 0.191 & 0.184 \\
\hline-0.447 & 0.303 & 0.296 & 0.287 & 0.280 & 0.271 & 0.264 \\
\hline-0.069 & 0.472 & 0.465 & 0.444 & 0.438 & 0.416 & 0.411 \\
\hline 0.196 & 0.603 & 0.596 & 0.560 & 0.562 & 0.534 & 0.527 \\
\hline 0.362 & 0.677 & 0.670 & 0.640 & 0.633 & 0.603 & 0.596 \\
\hline 0.823 & 0.825 & 0.818 & 0.788 & 0.781 & 0.751 & 0.744 \\
\hline 1.224 & 0.894 & 0.887 & 0.861 & 0.854 & 0.828 & 0.821 \\
\hline 1.955 & 0.951 & 0.945 & 0.926 & 0.920 & 0.901 & 0.894 \\
\hline 2.645 & 0.972 & 0.968 & 0.953 & 0.947 & 0.933 & 0.927 \\
\hline 3.311 & 0.982 & 0.979 & 0.966 & 0.962 & 0.950 & 0.945 \\
\hline
\end{tabular}

a $z=(\ln u) / 2 \sqrt{\sigma}$

where $\beta$ stands for

$$
\beta=N \sqrt{\sigma}
$$

When Eq. 13 is combined with Eq. $7 f_{N}$ can be obtained as a function of $z$ with $\sigma$ and $\beta$ as parameters. In Table I we compare approximate values of $f_{N}$ so obtained with the exact ones for $N=\infty, 1200$, and 600 , taking, as an example, the case where $\sigma$ is $2.0 \times 10^{-4}$. It is seen that Eq. 13 is a good approximation to the exact equation over essentially the entire range of helix-coil transition.

Alternation Frequency of Helical and Randomly Coiled Sections

At intermediate stages in the transition from coil to helix, a polypeptide molecule consists of alternating sequences of helical and randomly coiled sections. $\mathrm{Nagai}^{1}$ has given the exact expression for the number of alternating helical sections $g_{N}$ as a function of $N, u$, and $\sigma$. When treated subject to the conditions that $\sqrt{\sigma} \ll 1$, $N \gg 1$, and $N \sqrt{\sigma}>3$, his equation is eventually reduced to the form

$$
g_{N}=\beta \sqrt{f(1-f)}+(2 f-1) f
$$

The exact equations are also available ${ }^{1}$ for the distributions of length of helical and randomly coiled sections. We denote such a distribution function for helix by $L_{N}\left(n_{1}\right)$ and that for random coil by $M_{N}\left(n_{0}\right)$; thus $L_{N}\left(n_{1}\right)$ represents the number of helical sections each of which consists of $n_{1}$ monomeric residues, where $n_{1}$ can be varied from 3 to $N-2$. When approximated in accordance with the conditions mentioned above, Nagai's equation for $L_{N}\left(n_{1}\right)$ gives

$$
\begin{aligned}
& {\left[\frac{L_{N}\left(n_{1}\right)}{N \sigma}\right] \exp \left(-3 \sqrt{\sigma} \sqrt{\frac{1-f}{f}}\right)} \\
& =(1-f)\left(1-\frac{n_{1}}{N}+\frac{2 f}{\beta} \sqrt{\frac{f}{1-f}}\right) \\
& \times \exp \left(-\frac{n_{1}}{N} \beta \sqrt{\frac{1-f}{f}}\right) \\
& +\frac{f^{2}}{1-f}\left[1-\frac{n_{1}}{N}-\frac{2(1-f)}{\beta} \sqrt{\frac{1-f}{f}}\right] \\
& \times \exp \left[-\frac{\beta}{\sqrt{f(1-f)}}\left(1-\frac{n_{1}}{N} f\right)\right]
\end{aligned}
$$

The second term on the right-hand side of this expression becomes important only under the circumstances where $f$ is close to unity and $n_{1}$ approaches $N$. For indefinitely large $N$ Eq. 16 reduces to

$$
\begin{aligned}
& \lim _{N \rightarrow \infty}\left[L_{N}\left(n_{1}\right) / N\right]=\sigma(1-f) \\
& \times \exp \left[-\left(n_{1}-3\right) \sqrt{\sigma} \sqrt{\frac{1-f}{f}}\right]
\end{aligned}
$$

In a similar manner it can be shown that the corresponding approximation to $M_{N}\left(n_{0}\right)$ is given by 


$$
\begin{aligned}
M_{N}\left(n_{0}\right) / N \sigma=f\left[1-\frac{n_{0}}{N}-\frac{2}{\beta} \sqrt{f(1-f)}\right] \\
\quad \times \exp \left(-\frac{n_{0}}{N} \beta \sqrt{\frac{f}{1-f}}\right) \\
+f\left[1-\frac{n_{0}}{N}+\frac{2}{\beta} \sqrt{f(1-f)}\right] \\
\times \exp \left[-\frac{\beta}{\sqrt{f(1-f)}}+\frac{n_{0}}{N} \beta \sqrt{\frac{1-f}{f}}\right]
\end{aligned}
$$

and this reduces at the limit of infinite $N$ to

$$
\lim _{N \rightarrow \infty}\left[M_{N}\left(n_{0}\right) / N\right]=\sigma f \exp \left[-n_{0} \sqrt{\sigma} \sqrt{\frac{f}{1-f}}\right]
$$

Eliminating the intermediate variable $f$ from Eqs. 15, 16, and 18 with the aid of Eq. 13, we can determine $g_{N}, L_{N}\left(n_{1}\right)$, and $M_{N}\left(n_{0}\right)$ as functions of $f_{N}$, although this operation cannot be performed analytically. Also, substituting Eq. 7 into Eqs. 15, 16, and 18, these three quantities are expressed in terms of $z$ and hence of $\ln u$. Though not shown here, these approximate expressions have been found to agree closely with the corresponding exact equations when examined numerically for some typical cases of $\sigma$ and $N$.

\section{Molecular Dimensions}

In order to calculate the molecular dimensions of a polypeptide $\mathrm{Nagai}^{1}$ replaced the real polymer chain by the following configurational model. Here a helical section consisting of $n_{1}$ monomeric residues is replaced by a rod of $n_{1} b_{1}$ in length; a randomly coiled section consisting of $n_{0}$ residues is approximated by a random flight chain of $n_{0}$ steps each of which has a length $b_{0}$; and these two sections are connected alternatively at their ends by a universal joint. Miller and Flory ${ }^{3}$ performed a calculation by taking the steric effects on the conformation of randomly coiled chains into account, but their results cannot be subjected to the analytical treatment of deriving relevant approximate expressions for the molecular dimensions. It is our opinion that Nagai's model is capable of yielding information on the molecular dimensions of polypeptides which is accurate enough for practical purposes if a proper interpretation is made of the quantity $b_{0}$. However it is important to remember that neither Nagai's theory nor Miller-Flory's considers excluded volume effects on the randomly coiled sections.

Neglecting, as before, terms proportional to $\sqrt{\sigma}$ and $N^{-1}$ in comparison with unity and imposing the condition that $N \sqrt{\sigma}>3$ in order to ignore terms associated with the factor $\left(\lambda_{2} / \lambda_{1}\right)^{N}$, Nagai's result for $\left\langle R^{2}\right\rangle$, the mean-square end-to-end distance of the molecule, can be brought into the form

$$
\begin{aligned}
& \frac{\left\langle R^{2}\right\rangle}{N b_{0}^{2}(1-f)}=1+\frac{2 f}{\beta} \sqrt{\frac{f}{1-f}} \\
& +\frac{2}{\sqrt{\sigma}}\left(\frac{b_{1}}{b_{0}}\right)^{2}\left(\frac{f}{1-f}\right)^{3 / 2} q(\beta, f)
\end{aligned}
$$

where

$$
\begin{aligned}
& q(\beta, f)=1-\frac{3-2 J}{\beta} \sqrt{\frac{f}{1-f}} \\
& +\frac{1}{\beta f \sqrt{(1-f) f}} \exp \left(-\beta \sqrt{\frac{1-f}{f}}\right)
\end{aligned}
$$

At the limit of infinite $N, q$ tends to unity, and thus we have

$$
\lim _{N \rightarrow \infty} \frac{\left\langle R^{2}\right\rangle}{N b_{0}{ }^{2}(1-f)}=1+\frac{2}{\sqrt{\sigma}}\left(\frac{b_{1}}{b_{0}}\right)^{2}\left(\frac{f}{1-f}\right)^{3 / 2}
$$

which stands in perfect agreement with the equation derived by Ptitsyn $^{7}$ from a different approach.

To the degree of approximation in which Eq. 20 for $\left\langle R^{2}\right\rangle$ has been derived, Nagai's expression for $\left\langle S^{2}\right\rangle$, the mean-square radius of gyration of the molecule, is shown to take the form

$$
\begin{array}{r}
\frac{\left\langle S^{2}\right\rangle}{(1 / 6) N b_{0}{ }^{2}(1-f)}=1+\frac{12 f^{2}}{\beta^{2}}\left[1-\frac{2 \sqrt{f(1-f)}}{\beta}\right] \\
+\frac{2}{\sqrt{\sigma}}\left(\frac{b_{1}}{b_{0}}\right)^{2}\left(\frac{f}{1-f}\right)^{3 / 2} Q(\beta, f)
\end{array}
$$

where

$$
\begin{aligned}
& Q(\beta, f)=1-\frac{3}{\beta} \sqrt{\frac{f}{1-f}} \\
& \quad+\frac{6}{\beta^{2}}\left(\frac{f}{1-f}\right)\left[1-2(1-f)^{2}\right] \\
& \quad-\frac{6}{\beta^{3}}\left(\frac{f}{1-f}\right)^{3 / 2}\left[1-2(1-f)^{2}(3-2 f)\right. \\
& \left.\quad-\frac{(2 f-1)^{2}}{f^{4}} \exp \left(-\beta \sqrt{\frac{1-f}{f}}\right)\right]
\end{aligned}
$$


For infinitely large $N$ Eq. 23 reduces to

$$
\begin{aligned}
& \lim _{N \rightarrow \infty} \frac{\left\langle S^{2}\right\rangle}{(1 / 6) N b_{0}{ }^{2}(1-f)} \\
& \quad=1+\frac{2}{\sqrt{\sigma}}\left(\frac{b_{1}}{b_{0}}\right)^{2}\left(\frac{f}{1-f}\right)^{3 / 2}
\end{aligned}
$$

Comparison of Eqs. 22 and 25 gives the relation

$$
\left\langle R^{2}\right\rangle=6\left\langle S^{2}\right\rangle
$$

$\mathrm{Nagai}^{1}$ has shown that the rigorous expressions for infinite $N$ also satisfy this relation unless $f=1$.

Combining Eqs. 20 and 23 with Eq. 13, we can calculate $\left\langle R^{2}\right\rangle$ and $\left\langle S^{2}\right\rangle$ as functions of $f_{N}$ for fixed values of $\sigma, N, b_{0}$, and $b_{1}$. In Figure 1,

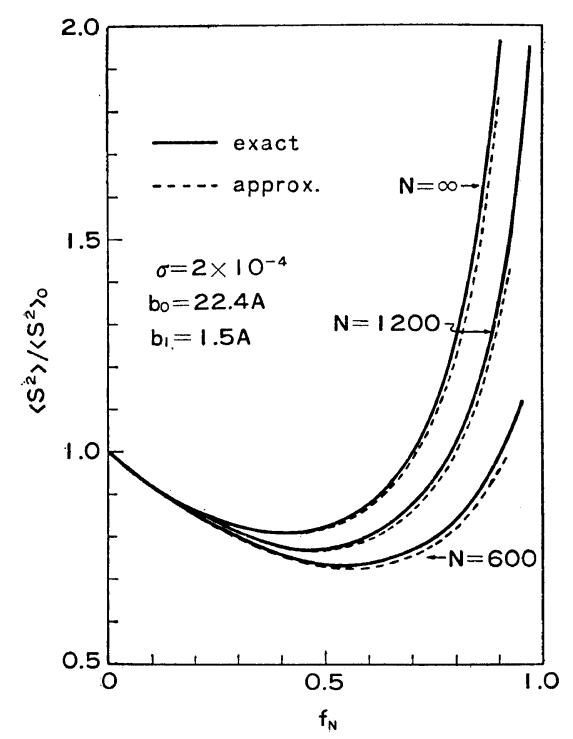

Figure 1. Comparison of the exact and approximate relations between $\left\langle S^{2}\right\rangle$ and $f_{N} ; \sigma$ is taken to be $2.0 \times 10^{-4}$.

the curves of $\left\langle S^{2}\right\rangle /(1 / 6) N b_{0}{ }^{2}$ vs. $f_{N}$ so calculated for $N=\infty, 1200$, and 600 with $\sigma=2.0 \times 10^{-4}$, $b_{0}=22.4 \mathrm{~A}$, and $b_{1}=1.5 \mathrm{~A}$ are compared with the values calculated from Nagai's exact expressions. It is seen that Eq. 23 can yield a fairly precise description of $\left\langle S^{2}\right\rangle$ over the entire range of helical content at least for the values of the parameters chosen. This degree of approxima- tion would be good enough for an analysis of experimental data.

Angular Dependence of the Intensity of Scattered Light

The particle scattering function $P(\theta)$ for a polypeptide molecule in solution must be evaluated by taking into account the copolymer nature of the molecule, which stems from the fact that the specific refractive index increment of its helical sections, $(\partial n / \partial c)_{1}$, may be different from that of its randomly coiled sections, $(\partial n / \partial c)_{0}$. According to Nagai, ${ }^{1}$ the reciprocal of $P(\theta)$ can be expanded in powers of $k^{2}$ as

$$
\boldsymbol{P}(\theta)^{-1}=1+\frac{k^{2}}{3}\left\langle S^{2}\right\rangle_{a p p}+0\left(k^{4}\right)
$$

where $k$ denotes $(4 \pi / \lambda) \sin (\theta / 2)$, with $\lambda$ and $\theta$ being the wavelength of the incident beam in the solution considered and the angle between the scattered and incident beams of light, respectively. The quantity $\left\langle S^{2}\right\rangle_{a p p}$, which may be termed the apparent mean-square radius of gyration, is shown to be expressed by the equation

$$
\begin{aligned}
& \left\langle S^{2}\right\rangle_{a p p}=\left\langle S^{2}\right\rangle\left(1+\delta f_{N}\right)^{-2} \\
& \times\left\{1+\frac{\delta}{N^{2}\left\langle S^{2}\right\rangle} \sum_{i<j}\left[2\left\langle{r_{i j}}^{2}\right\rangle_{10}+(2+\delta)\left\langle r_{i j}{ }^{2}\right\rangle_{11}\right]\right\}
\end{aligned}
$$

Here

$$
\delta=(\partial n / \partial c)_{1} /(\partial n / \partial c)_{0}-1
$$

$\left\langle r_{i j}{ }^{2}\right\rangle_{11}$ is the mean-square distance between monomer $i$ and monomer $j$ when they both belong to either the same or different helical sections in a given polypeptide chain, and $\left\langle\boldsymbol{r}_{i j}\right\rangle_{10}$ is the value of $\left\langle r_{i j}{ }^{2}\right\rangle$ when monomer $i$ belongs to a helical section and monomer $j$ to a randomly coiled section, where the monomers are numbered $1,2, \ldots, N$ from one end to the other of the chain. In deriving Eq. 28, use has been made of the relation

$$
\left\langle S^{2}\right\rangle=N^{-2} \sum_{i<j}\left(\left\langle r_{i j}{ }^{2}\right\rangle_{11}+\left\langle r_{i j}{ }^{2}\right\rangle_{00}+2\left\langle r_{i j}{ }^{2}\right\rangle_{10}\right)
$$

where the meaning of $\left\langle r_{i j}{ }^{2}\right\rangle_{00}$ is self-explanatory. Now, we have

$\sum_{i<j}\left\langle r_{i j}{ }^{2}\right\rangle_{11}=J_{1}(1,1)+2\left[J_{2}(1,1)+J_{3}(1,1)\right]$

where 
A. Teramoto, T. Norisuye, and H. Fujita

$$
\begin{aligned}
& J_{1}(1,1)=\sum_{2 \leq i<s \leq j \leq N-1}\left\langle\boldsymbol{r}_{s}^{2}\right\rangle_{11} \\
& J_{2}(1,1)=\sum_{2 \leq i<s \leq j-1 \leq N-2}\left\langle\boldsymbol{r}_{s} \cdot \boldsymbol{r}_{s+1}\right\rangle_{11} \\
& J_{3}(1,1)=\sum_{2 \leq i<s<t-1 \leq j-1 \leq N-2}\left\langle\boldsymbol{r}_{s} \cdot \boldsymbol{r}_{t}\right\rangle_{11}
\end{aligned}
$$

The quantity $\boldsymbol{r}_{i}$ denotes the displacement vector of monomer $i$. If we denote by $\mu_{i}$ the probability that monomer $i$ is in a helical section, Eq. 31 may be written

$$
\begin{aligned}
& J_{1}(1,1) \\
& =b_{0}{ }^{2} \sum_{2 \leq i<s \leq j \leq N-1}\left[\left\langle\mu_{i} \mu_{j}\right\rangle-\left(1-\frac{b_{1}{ }^{2}}{{b_{0}}^{2}}\right)\left\langle\mu_{i} \mu_{s} \mu_{j}\right\rangle\right]
\end{aligned}
$$

where $b_{0}$ and $b_{1}$ have the same meanings as defined above. The expressions for $\left\langle\mu_{i} \mu_{j}\right\rangle$ and $\left\langle\mu_{i} \mu_{s} \mu_{j}\right\rangle$ can be derived from Nagai's theory, yielding

$$
\begin{aligned}
& \left\langle\mu_{i} \mu_{j}\right\rangle \\
& =Z_{N}{ }^{-1}(u \sigma)^{-1} \sum_{m, n, p} c\left(\lambda_{m}\right) c\left(\lambda_{n}\right) c\left(\lambda_{p}\right) \lambda_{m}{ }^{i-1} \lambda_{n}{ }^{j-i-2} \lambda_{p}{ }^{N-j} \\
& \quad \times\left(\lambda_{m}-u\right)\left(\lambda_{p}-u\right)\left[\lambda_{m} \lambda_{n}+\lambda_{m}\left(\lambda_{m}-u\right)+\lambda_{n}\right. \\
& \left.\quad \times\left(\lambda_{n}-u\right)\right]\left[\lambda_{n} \lambda_{p}+\lambda_{n}\left(\lambda_{n}-u\right)+\lambda_{p}\left(\lambda_{p}-u\right)\right] \\
& \left\langle\mu_{i} \mu_{s} \mu_{j}\right\rangle=Z_{N}{ }^{-1}(u \sigma)^{-1} \sum_{m, n, p, q} c\left(\lambda_{m}\right) c\left(\lambda_{n}\right) c\left(\lambda_{p}\right) c\left(\lambda_{q}\right) \\
& \quad \times \lambda_{m}{ }^{i-1} \lambda_{n}{ }^{s-i-2} \lambda_{p}{ }^{j-s-2} \lambda_{q}{ }^{N-j}\left(\lambda_{m}-u\right)\left(\lambda_{q}-u\right) \\
& \quad \times\left[\lambda_{m} \lambda_{n}+\lambda_{m}\left(\lambda_{m}-u\right)+\lambda_{n}\left(\lambda_{n}-u\right)\right] \\
& \quad \times\left[\lambda_{n} \lambda_{p}+\lambda_{n}\left(\lambda_{n}-u\right)+\lambda_{p}\left(\lambda_{p}-u\right)\right] \\
& \quad \times\left[\lambda_{p} \lambda_{q}+\lambda_{p}\left(\lambda_{p}-u\right)+\lambda_{q}\left(\lambda_{q}-u\right)\right]
\end{aligned}
$$

Here the reader is referred to the original article of $\mathrm{Nagai}^{1}$ as for the definitions of $Z_{N}$ and $c\left(\lambda_{i}\right)$ $(i=1,2,3,4)$. In a similar way, it can be shown that

$$
\begin{aligned}
& J_{2}(1,1)=b_{1}{ }^{2} \sum_{2 \leq i<s \leq j-1 \leq N-2}\left\langle\mu_{i} \mu_{s} \mu_{s+1} \mu_{j}\right\rangle \\
& J_{3}(1,1)=b_{1}{ }^{2} \sum_{2 \leq i<s<t-1 \leq j-1 \leq N-2}\left\langle\mu_{i} \mu_{s} \mu_{s \cdot 1} \cdots \mu_{t} \mu_{j}\right\rangle
\end{aligned}
$$

with

$$
\begin{aligned}
&\left\langle\mu_{i} \mu_{s} \mu_{s+1} \mu_{j}\right\rangle \\
&= Z_{N}{ }^{-1}(u \sigma)^{-1} \sum_{m, n, p, q} c\left(\lambda_{m}\right) c\left(\lambda_{n}\right) c\left(\lambda_{p}\right) c\left(\lambda_{q}\right) \\
& \times \lambda_{m}^{i-1} \lambda_{n}^{s-i-1} \lambda_{p}^{j-s-2} \lambda_{q}{ }^{N-j} \\
& \times\left(\lambda_{m}-u\right)\left(\lambda_{q}-u\right)\left(\lambda_{n}+\lambda_{p}-u\right) \\
& \times\left[\lambda_{m} \lambda_{n}+\lambda_{m}\left(\lambda_{m}-u\right)+\lambda_{n}\left(\lambda_{n}-u\right)\right] \\
& \times\left[\lambda_{p} \lambda_{q}+\lambda_{p}\left(\lambda_{p}-u\right)+\lambda_{q}\left(\lambda_{q}-u\right)\right]
\end{aligned}
$$

$$
\begin{aligned}
&\left\langle\mu_{i} \mu_{s} \mu_{s+1} \cdots \mu_{t} \mu_{j}\right\rangle \\
&= Z_{N}{ }^{-1}(u \sigma)^{-1} u^{t-s-2} \sum_{m, n, p, q} c\left(\lambda_{m}\right) c\left(\lambda_{n}\right) c\left(\lambda_{p}\right) c\left(\lambda_{q}\right) \\
& \times \lambda_{m}{ }^{i-1} \lambda_{n}{ }^{s-i} \lambda_{p}{ }^{j-t} \lambda_{q}{ }^{N-j}\left(\lambda_{m}-u\right)\left(\lambda_{q}-u\right) \\
& \times\left[\lambda_{m} \lambda_{n}+\lambda_{m}\left(\lambda_{m}-u\right)+\lambda_{n}\left(\lambda_{n}-u\right)\right] \\
& \times\left[\lambda_{p} \lambda_{q}+\lambda_{p}\left(\lambda_{p}-u\right)+\lambda_{q}\left(\lambda_{q}-u\right)\right]
\end{aligned}
$$

Writing

$$
\sum_{i<j}\left\langle r_{i j}^{2}\right\rangle_{10}=J_{1}(1,0)+2\left[J_{2}(1,0)+J_{3}(1,0)\right]
$$

with $J_{1}(1,0) J_{2}(1,0)$, and $J_{3}(1,0)$ defined as

$$
\begin{aligned}
& J_{1}(1,0)=\sum_{2 \leq i<s \leq j \leq N}\left\langle\boldsymbol{r}_{s}{ }^{2}\right\rangle_{10} \\
& J_{2}(1,0)=\sum_{2 \leq i<s \leq j-2 \leq N-2}\left\langle\boldsymbol{r}_{s} \cdot \boldsymbol{r}_{s+1}\right\rangle_{10} \\
& J_{3}(1,0)=\sum_{2 \leq i<s<t-1 \leq j-2 \leq N-2}\left\langle\boldsymbol{r}_{s} \cdot \boldsymbol{r}_{t}\right\rangle_{10}
\end{aligned}
$$

we find that

$$
\begin{aligned}
J_{1}(1,0) & =b_{0}{ }^{2} \sum_{2 \leq i<s \leq j \leq N}\left\{\left\langle\mu_{i}\right\rangle-\left(1-\frac{b_{1}{ }^{2}}{b_{0}{ }^{2}}\right)\left\langle\mu_{i} \mu_{s}\right\rangle\right. \\
& \left.-\left[\left\langle\mu_{i} \mu_{j}\right\rangle-\left(1-\frac{b_{1}{ }^{2}}{b_{0}{ }^{2}}\right)\left\langle\mu_{i} \mu_{s} \mu_{j}\right\rangle\right]\right\} \quad \text { (45) } \\
J_{2}(1,0) & =b_{1}{ }^{2}{ }^{2 \leq i<s \leq j-2 \leq N-2}{ }\left[\left\langle\mu_{i} \mu_{s} \mu_{s+1}\right\rangle\right. \\
& \left.-\left\langle\mu_{i} \mu_{s} \mu_{s+1} \mu_{j}\right\rangle\right] \\
J_{3}(1,0) & =b_{1}{ }^{2}{ }_{2 \leq i<s<t-1 \leq j-2 \leq N-2}\left[\left\langle\mu_{i} \mu_{s} \mu_{s+1} \cdots \mu_{t}\right\rangle\right. \\
& \left.-\left\langle\mu_{i} \mu_{s} \mu_{s+1} \cdots \mu_{t} \mu_{j}\right\rangle\right]
\end{aligned}
$$

where

$$
\begin{aligned}
&\left\langle\mu_{i}\right\rangle=Z_{N}{ }^{-1}(u \sigma)^{-1} \sum_{m, n} c\left(\lambda_{m}\right) c\left(\lambda_{n}\right) \lambda_{m}{ }^{i-1} \lambda_{n}{ }^{N-i} \\
& \times\left(\lambda_{m}-u\right)\left(\lambda_{n}-u\right) \\
& \times\left[\lambda_{m} \lambda_{n}+\lambda_{m}\left(\lambda_{m}-u\right)+\lambda_{n}\left(\lambda_{n}-u\right)\right] \\
&\left\langle\mu_{i} \mu_{s} \mu_{s+1}\right\rangle=Z_{N}{ }^{-1}(u \sigma)^{-1} \sum_{m, n, v} c\left(\lambda_{m}\right) c\left(\lambda_{n}\right) c\left(\lambda_{p}\right) \\
& \quad \times \lambda_{m}{ }^{i-1} \lambda_{n}{ }^{s-i-1} \lambda_{p}^{N-s} \\
& \quad \times\left(\lambda_{m}-u\right)\left(\lambda_{p}-u\right)\left(\lambda_{n}+\lambda_{p}-u\right) \\
& \quad \times\left[\lambda_{m} \lambda_{n}+\lambda_{m}\left(\lambda_{m}-u\right)+\lambda_{n}\left(\lambda_{n}-u\right)\right]
\end{aligned}
$$

$$
\left\langle\mu_{i} \mu_{s} \mu_{s+1} \cdots \mu_{t}\right\rangle
$$

$$
\begin{aligned}
= & Z_{N}{ }^{-1}(u \sigma)^{-1} u^{t-s-2} \sum_{m, n, p} c\left(\lambda_{m}\right) c\left(\lambda_{n}\right) c\left(\lambda_{p}\right) \\
& \times \lambda_{m}{ }^{i-1} \lambda_{n}{ }^{8-i} \lambda_{p}{ }^{N-t+2}\left(\lambda_{m}-u\right)\left(\lambda_{p}-u\right) \\
& \times\left[\lambda_{m} \lambda_{n}+\lambda_{m}\left(\lambda_{m}-u\right)+\lambda_{n}\left(\lambda_{n}-u\right)\right]
\end{aligned}
$$

We calculated $J_{1}(1,1), \ldots J_{3}(1,0)$ subject to the previously imposed conditions that $\sqrt{\sigma} \ll 1, N^{-1}$ $\ll 1$, and $N \sqrt{\sigma}>3$, with the summations being 
replaced by integrals. The results obtained were yet so complicated with terms multiplied by $\exp [-\beta \sqrt{(1-f) / f}]$ that we had to be content with the case where this exponential function could be taken to be negligible in comparison with unity. The resulting expression for $\left\langle S^{2}\right\rangle_{a p p}$ then reads

$$
\begin{aligned}
& \left\langle S^{2}\right\rangle_{a p p} /\left\langle S^{2}\right\rangle=\left(1+\delta f_{N}\right)^{-2} \\
\times & \left\{(1+\delta f)^{2}+\left[(\delta f)^{2} K_{1}+2 \delta f K_{2}\right] \frac{(1-f) N b_{0}{ }^{2}}{6\left\langle S^{2}\right\rangle}\right\}
\end{aligned}
$$

where

$$
\begin{aligned}
& K_{1}=I_{1}(\beta, f)+\frac{2}{\sqrt{\sigma}}\left(\frac{b_{1}}{b_{0}}\right)^{2}\left(\frac{f}{1-f}\right) P_{1}(\beta, f) \\
& K_{2}=I_{2}(\beta, f)+\frac{2}{\sqrt{\sigma}}\left(\frac{b_{1}}{b_{0}}\right)^{2}\left(\frac{f}{1-f}\right) P_{2}(\beta, f)
\end{aligned}
$$

with

$$
\begin{aligned}
I_{1} & =-\frac{12}{\beta} \sqrt{f(1-f)} \\
& \times\left[1-\frac{1}{\beta}(5-6 f) \sqrt{\frac{f}{1-f}}+\frac{2}{\beta^{2}} f(5-6 f)\right]
\end{aligned}
$$

$$
\begin{aligned}
I_{2} & =-\frac{6}{\beta} \sqrt{f(1-f)} \\
& \times\left[1-\frac{1}{\beta}(3-4 f) \sqrt{\frac{f}{1-f}}+\frac{4}{\beta^{2}} f(1-f)\right]
\end{aligned}
$$

$$
\begin{aligned}
P_{1} & =\frac{6}{\beta}\left\{1-2 f+\frac{1}{\beta} \sqrt{\frac{1-f}{f}}\right. \\
& \times\left[1-8 f+10 f^{2}-\frac{2 f}{1-f}\left(1-3 f+f^{2}\right)\right] \\
& -\frac{1}{\beta^{2}}\left[(1-f)\left(2-6 f+5 f^{2}\right)+f\left(9-4 f^{2}\right)\right. \\
& \left.\left.+\frac{2 f(2 f-1)}{1-f}\right]\right\} \\
P_{2} & =\frac{3}{\beta}\left\{1-2 f-\frac{2}{\beta}\left(3-8 f+4 f^{2}\right) \sqrt{\frac{f}{1-f}}\right. \\
& \left.+\frac{2}{\beta^{2}}\left[2 f\left(2-5 f+2 f^{2}\right)+\frac{f(1-2 f)}{1-f}\right]\right\}
\end{aligned}
$$

For the additional condition imposed above to be satisfied it would be sufficient if we take such that $\beta \sqrt{(1-f) / f}>5$. Thus even if we set $\beta>10$, the values of $f$ for which Eq. 51 is useful must be confined to values smaller than 0.8 .

When $\delta$ is zero, Eq. 51 gives $\left\langle S^{2}\right\rangle_{a p p}=\left\langle S^{2}\right\rangle$, as should be expected. In the limit where $N=\infty$, $K_{1}$ and $K_{2}$ tend to zero, $f_{N}$ approaches $f$, and $\left\langle S^{2}\right\rangle / N$ remains finite (see Eq. 25), so that $\left\langle S^{2}\right\rangle_{a p p}$ becomes equal to $\left\langle S^{2}\right\rangle$. Thus for polypeptide samples of sufficiently high molecular weight the measured values of $\left\langle S^{2}\right\rangle_{a p p}$ may be taken equal to the true values of $\left\langle S^{2}\right\rangle$, irrespective of their helical content as well as the difference in refractive index increment between helical and randomly coiled sections. In order to know the behavior of $\left\langle S^{2}\right\rangle_{a p p}$ for finite $N$ and nonzero $\delta$ we calculated the values of this quantity for a number of $f$ in the range $0.1<f<0.9$ by assigning the following numerical data to Eq. 51: $\delta=0.2{ }^{*} b_{0}=12 \mathrm{~A}, b_{1}=1.5 \mathrm{~A}, \sigma=10^{-4}$, and $N=1000$ (thus $\beta=10$ ). The resulting values of $\left\langle S^{2}\right\rangle_{a p p}$ agreed with the corresponding $\left\langle S^{2}\right\rangle$ to within $1 \%$, at all $f$ examined. Additional calculations with $\delta=0.4$ did not give $\left\langle S^{2}\right\rangle_{a p p}$ which exceeded $\left\langle S^{2}\right\rangle$ more than $2 \%$. From these results it appears quite safe to conclude that except under very special circumstances the slope of $P(\theta)^{-1} v s$. $k^{2} / 3$ for a polypeptide sample gives a quantity which is essentially equal to its mean-square radius of gyration even when the molecule is in the state of interrupted helix.

\section{DISCUSSION}

As has been shown in Part VI, Eq. 13 can be utilized to evaluate the parameter $u$ and $\sigma$ when data for $f_{N}$ as a function of $N$ are available. This method involves no restriction other than $\sqrt{\sigma} \ll 1$ and $N \sqrt{\sigma}>3$, and is equally applicable to both thermally induced and solvent-induced transitions of a polypeptide from randomly coiled to helical state. Therefore it is now possible to investigate experimentally how $u$ and $\sigma$ vary with environmental conditions such as temperature, pressure, solvent species, and solvent composition. Once $\sigma$ is known, we can calculate $g_{N}, L_{N}\left(n_{1}\right)$, and $M_{N}\left(n_{0}\right)$ as functions of $f_{N}$ from Eqs. 15, 16,

* It has been demonstrated in Part VI that the value of $\delta$ for poly- $\mathrm{N}^{5}$-(3-hydroxypropyl)-L-glutamine ranged between 0.2 and 0.4 . 


\section{A. Teramoto, T. Norisuye, and H. Fujita}

and 18 combined with Eq. 13. These approximate expressions allow the desired numerical calculations to carry through much faster than do the original exact ones. The results enable us to characterize quantitatively what conformation the polypeptide molecule assumes at intermediate stages of its transition from coil to helix. In other words, quantitative information is obtained about the molecular shape which is often ambiguously termed interrupted helix. Such information is fundamental in correlating the size and shape of a polypeptide molecule with its flow properties such as limiting viscosity number, sedimentation coefficient, flow birefringence, etc.

The first two terms on the right-hand side of Eq. 23 are the contributions from randomly coiled sections and the last term from helical sections. At fixed $f$ and $\beta$ the relative importance of the two contributions is determined by a combined parameter $(1 / \sqrt{\sigma})\left(b_{1} / b_{0}\right)^{2}$. The merit of Eq. 23 is not only in its form which permits a straightforward computation of $\left\langle S^{2}\right\rangle$ but also in the fact that it can be used to determine the parameters $b_{0}$ and $b_{1}$ when experimental data are available for (a) $N$ of the sample, (b) $\left\langle S^{2}\right\rangle$ as a function of $f_{N}$, and (c) $\sigma$ of the system. This determination may be carried through in the following way. First substitute the known values of $N$ and $\sigma$ into Eq. 13 and calculate $f$ corresponding to $f_{N}$ for which the measured values of $\left\langle S^{2}\right\rangle$ are given. Then, with these data for $f$ and the value of $\beta$ calculated from the given $N$ and $\sigma$, compute

$$
\begin{aligned}
& H_{1}=\left\langle S^{2}\right\rangle /(1 / 6) N(1-f) \\
& H_{2}=1+\frac{12 f^{2}}{\beta^{2}}\left[1-\frac{2 \sqrt{f(1-f)}}{\beta}\right]
\end{aligned}
$$

and

$$
H_{3}=\left(\frac{f}{1-f}\right)^{3 / 2} Q(\beta, f)
$$

as functions of $f$, and plot $H_{1} / H_{2}$ against $\mathrm{H}_{3} / \mathrm{H}_{2}$ corresponding to the same $f$. Eq. (23) indicates that this plot should be linear and its ordinate intercept and slope are equal to $b_{0}{ }^{2}$ and $2 b_{1}{ }^{2} / \sqrt{\sigma}$, respectively. Thus the values of $b_{0}$ and $b_{1}$ are obtained by finding a straight line which fits $H_{1} / H_{2}$ plotted against $H_{3} / H_{2}$.

One of the central problems in the study of dilute polymer solutions is to investigate how the limiting viscosity number $[\eta]$ is related to the size and shape of polymer molecules. For polypeptides taking the shape termed interrupted helix this problem yet remains unsolved. Ptitsyn ${ }^{7}$ assumed that the Flory-Fox relation, common to randomly coiled polymers, is also applicable between $[\eta]$ and $\left\langle R^{2}\right\rangle$ of interrupted helices and for finite $N$ used in Eq. 22 by replacing $f$ with $f_{N}$. Evidently, it is more reasonable to state the FloryFox relation in terms of $\left\langle S^{2}\right\rangle$ rather than $\left\langle R^{2}\right\rangle$, because $\left\langle S^{2}\right\rangle$ is expected to be a more relevant measure for the size of a polymer molecule not only of the randomly coiled type but also of the interrupted-helix type. However, so far as the validity of the Flory-Fox relation is concerned, we can say nothing conclusive until simultaneous measurements of $\left\langle S^{2}\right\rangle$ and [ $\left.\eta\right]$ are undertaken over the entire region of helix-coil transition of a polypeptide. The use of Eq. 22 for $\left\langle R^{2}\right\rangle$ or its equivalent Eq. 25 for $\left\langle S^{2}\right\rangle$ for finite $N$ can be checked in terms of the approximate expression derived above. Such a test with respect to $\left\langle S^{2}\right\rangle$ is shown in Figure 2. Here the solid lines represent the relations between $\left\langle S^{2}\right\rangle /(1 / 6) N b_{0}{ }^{2}\left(1-f_{N}\right)$ and $\left[f_{N} /\left(1-f_{N}\right)\right]^{3 / 2}$ for $N=\infty, 1200$, and 600 calculated from eqs. (13) and (23) with $\sigma=2.0 \times$ $10^{-4}$ and $\left(b_{1} / b_{0}\right)^{2}=(1.5 / 22.4)^{2}$. If Ptitsyn's treatment is valid, these plots for all $N$ should follow

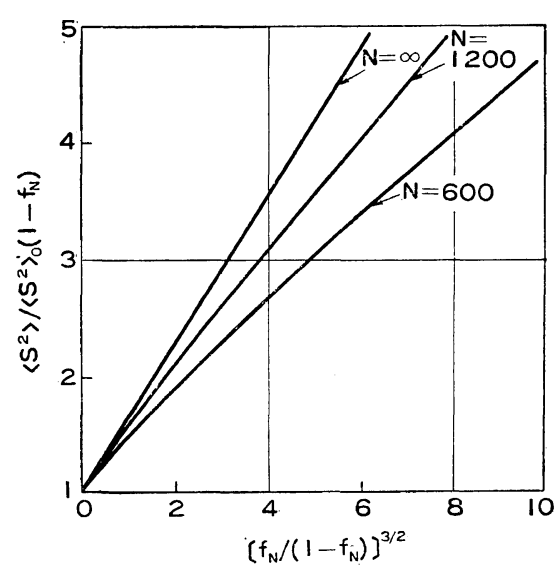

Figure 2. Plots of $\frac{\left\langle S^{2}\right\rangle}{\left\langle S^{2}\right\rangle_{0}\left(1-f_{N}\right)}$ against $\left(\frac{f_{N}}{1-f_{N}}\right)^{3 / 2}$ as a function of $N$. $\left\langle S^{2}\right\rangle_{0}$ stands for $(1 / 6) N b_{0}^{2}$. 
the solid line which corresponds to $N=\infty$. The marked divergence of the lines for finite $N$ from this line indicates that Eq. 25 for $\left\langle S^{2}\right\rangle$ is not applicable for samples of finite $N$. The same is shown to be true of $\left\langle R^{2}\right\rangle$.

The approximate treatment developed in this paper may be extended to other cases of interest, for example, to the calculation of $\left\langle r_{i j}{ }^{4}\right\rangle$ and higher moments of $r_{i j}$. We plan to perform such a calculation in connection with the experimental determination of $\left\langle S^{2}\right\rangle$ of typical polypeptides by light scattering.

Acknowledgment. We wish to thank Dr. K. Nagai for his interest in this investigation. Acknowledgment is made for financial support from the Ministry of Education.

\section{REFERENCES}

1. K. Nagai, J. Chem. Phys., 34, 887 (1961).

2. K. Okita, A. Teramoto, and H. Fujita, Biopolymers, in press.

3. W. G. Miller and P. J. Flory, J. Mol. Biol., 15, 298 (1966).

4. M. Go, N. Saito, and M. Ochiai, J. Phys. Soc. Japan, 22, 227 (1967).

5. O.B. Ptitsyn and A.M. Skvortsev, Biofizika, 10, 909 (1965).

6. B. H. Zimm and J. K. Bragg, J. Chem. Phys., 31, 526 (1959).

7. O. B. Ptitsyn, Conformation of Biopolymers, G. N. Ramachandran, Ed., (Academic Press, Inc., New York, 1967) Vol. 1, p. 381. 